\title{
Le concept de maladies virales émergentes : quel risque de zoonose pour La Réunion?
}

\author{
The concept of emerging viral diseases: what risk for Reunion island?
}

\author{
M. Peton · P. Vilain · O. Reilhes $\cdot$ E. Cardinale $\cdot$ B.A. Gaüzère $\cdot$ L. Filleul \\ Reçu le 27 avril 2012; accepté le 16 avril 2013 \\ (C) Société de pathologie exotique et Springer-Verlag France 2013
}

\begin{abstract}
Résumé A La Réunion, le risque d'émergence de maladies virales est constitué par plusieurs zoonoses virales qu'il convient de surveiller : infections à virus Sindbis, virus de l'encéphalite japonaise, virus Wesselsbron, virus Nipah, virus Zika, virus West Nile, virus de la fièvre de la vallée du Rift. La lutte contre ces maladies virales émergentes (MVE) passe par une détection précoce des cas et donc un système de surveillance doté d'un véritable réseau d'information, d'alerte et de prévention international.
\end{abstract}

Mots clés Maladies virales émergentes · Dynamique Potentiel épidémique $\cdot$ Surveillance $\cdot$ Réseau d'information international $\cdot$ Zoonoses $\cdot$ Virus Sindbis · Virus de l'encéphalite japonaise · Virus Wesselsbron · Virus Nipah · Virus Zika · Virus West Nile · Virus de la fièvre de la vallée du Rift · Océan indien · La Réunion

M. Peton · P. Vilain $\cdot$ L. Filleul $(\bowtie)$

Cellule de l'Institut de veille sanitaire en région océan Indien, Institut de veille sanitaire, Saint-Denis, La Réunion, France

e-mail : laurent.filleul@ars.sante.fr

O. Reilhes

Service de lutte anti-vectorielle de l'Agence

de santé océan Indien, délégation Réunion, Saint-Denis,

La Réunion, France

E. Cardinale

Centre de coopération internationale pour

la recherche agronomique pour le développement (CIRAD),

UMR CMAEE Saint Denis, La Réunion, France

Centre de recherche et de veille sur les maladies émergentes dans l'océan Indien (CRVOI), Saint Denis, La Réunion, France

B.A. Gaüzère

CHU de La Réunion, site Centre hospitalier Félix Guyon,

Saint Denis, La Réunion, France

Centre René Labusquière, Université Bordeaux Segalen, Bordeaux, France
Abstract In Reunion Island, the risk of emerging infectious diseases lies mainly in several viral zoonoses: West Nile fever, Sindbis virus, Nipah virus, Wesselsbron virus, Rift Valley fever and Japanese encephalitis. There morbidity and consequences are more or less important but they all have a non-negligible epidemic potential, so they have to be monitored. Indeed, the struggle against these emerging infectious diseases requires an early detection of the cases, thus a surveillance system capable of detecting them as early as possible, thanks to a real international network of information, warning and prevention.

Keywords Emerging infectious diseases - Dynamics · Epidemics potential $\cdot$ Watch $\cdot$ International information network $\cdot$ Zoonosis $\cdot$ Sindbis virus $\cdot$ Japanese encephalitis virus · Wesselsbron Virus · Nipah Virus · West Nile virus · Rift valley virus $\cdot$ Indian Ocean $\cdot$ Reunion island

\section{Introduction}

\section{L'océan Indien, une zone sous influence}

L'océan Indien est une zone qui répond aux conditions d'émergence des maladies virales émergentes (MVE) [9] : climat tropical chaud et humide, présence d'une grande variété de vecteurs, forts indices vectoriels, circulation intense des biens et des personnes entre les pays et les continents : Afrique, Europe, Asie, Australie.

Les populations insulaires de la zone sont immunologiquement naïves vis-à-vis de nombreuses maladies infectieuses potentiellement émergentes en raison de l'absence de circulation autochtone intense des agents pathogènes ou de vague épidémique récente dans les îles.

Les inégalités entre les pays de l'océan Indien en termes de système de santé, d'accès aux soins, de réseau de surveillance, d'accès à des conditions d'hygiène et de nutrition correctes, sont des facteurs favorables à l'émergence de 
certaines MVE. Les échanges entre les pays favorisent ensuite leur dissémination rapide quels que soient les niveaux de développement et de richesse des pays touchés.

D'autre part, le climat tropical chaud et humide du sudouest de l'océan Indien, tout particulièrement du mois de décembre au mois d'avril, est propice à la présence et au développement de vecteurs (moustiques) et de réservoirs (certaines espèces animales) des maladies infectieuses.

Les pays limitrophes de l'océan Indien d'Afrique (Afrique du Sud, Kenya, Mozambique, Somalie, Tanzanie), du Moyen Orient (Iran, Oman, Yémen), d'Asie (Bengladesh, Birmanie, Inde, Indonésie, Maldives Pakistan, Sri Lanka, Thailande), l'Australie, les Comores, Madagascar, Maurice, La Réunion et les Seychelles regroupent plus de deux milliards d'habitants, sans compter la Chine et l'Inde dans lesquelles se rendent très régulièrement les Réunionnais originaires de ces territoires.

En outre, les changements climatiques, technologiques et environnementaux augmentent dans toute la région (activité de l'Homme sur l'environnement, inondations, sécheresse, modification écologique) entraînant des conditions propices à la diffusion potentielle de vecteurs et d'hôtes infectieux.

De plus, il existe au sein de l'océan Indien et dans son périmètre de nombreux agents infectieux.

Certains d'entre eux sont latents, tel le virus Zika qui ne présente pas de caractère zoonotique évident car, hormis l'Homme, son seul hôte vertébré connu à ce jour est le singe Macacca mulatta,. D'autres sont présents uniquement chez les animaux, tel le virus Wesselsbron.

D'autres virus qui ont un caractère zoonotique plus marqué ont été signalés dans le pourtour de l'océan Indien ou dans des régions dont est originaire une partie de la population réunionnaise.

Les virus Tembusu [4] et Baiyangdian dont un des vecteurs est Culex tritaeniorhynchus ont ravagé, en 2010, de nombreux élevages industriels de canards en Chine, induisant de fortes pertes économiques ; le virus Sitiawan [12] a été responsable d'encéphalites dans des élevages de poulets en Malaisie ; le virus Bagaza [1] également isolé de Culex tritaeniorhynchus qui est très proche sinon identique au virus Turkey Meningo encephalitis, est un agent de zoonose aviaire qui a provoqué des encéphalites humaines dans le sud de l'Inde (Kerala) ; le virus Hendra [8] s'est rendu responsable d'encéphalites humaines et équines en Australie. Des anticorps spécifiques contre les virus Nipah, Hendra et Tioman ont été retrouvés chez des chauves-souris à Madagascar [13].

À une bien plus grande distance de La Réunion, le virus Usutu a été isolé d'Aedes albopictus en Italie du nord [3], or ce vecteur abonde à La Réunion. Ce virus, qui s'est solidement et rapidement implanté dans l'avifaune sauvage d'une grande partie de l'Europe occidentale, peut également infecter le Cheval et l'Homme, chez lequel il a provoqué au moins deux infections neuro-invasives fatales.
Enfin, d'autres virus ont déjà confirmé leur virulence au cours d'épidémies de grande ampleur tel le Chikungunya qui a touché l'océan Indien en 2005 et 2006. Cette épidémie partie du Kenya en 2004 s'est propagée aux pays du sudouest de l'océan Indien en 2005, avant de gagner l'Inde en 2007 [16].

\section{La situation à La Réunion}

La Réunion n'échappe pas à ces particularités et le risque d'émergence y est bien présent. À partir de la littérature, nous avons identifié plusieurs zoonoses virales à risque d'émergence à La Réunion en fonction des facteurs « hôte », « environnement » et " agent », afin d'établir une surveillance ciblée.

Ces zoonoses sont les infections à virus Sindbis, Nipah, West Nile, Wesselsbron, l'encéphalite japonaise qui à partir de 1995, a gagné le nord-est de l'Australie, et la fièvre de la vallée du Rift (FVR). Leurs principales caractéristiques sont résumées dans le tableau 1 .

Il convient de souligner que cette liste n'est pas exhaustive, car de nouvelles émergences se font jour régulièrement en périphérie de l'océan Indien : les infections à virus Tembusu et Baiyangdian, à virus Sitiawan, à virus Bagaza, à virus Hendra et à virus Usutu, en sont des exemples.

\section{Des hôtes susceptibles}

\section{Présence de certains hôtes}

Il existe à La Réunion environ 520 élevages de porcs (pour une production annuelle d'environ 12000 tonnes et une couverture du marché de près de $50 \%$ ) et de nombreux élevages familiaux de cabris qui échappent aux contrôles vétérinaires pour des raisons religieuses. La filière bovine comprend quant à elle environ 315 élevages, pour un taux de couverture du marché de $32 \%$ [6]. Ces animaux sont les hôtes naturels potentiels des virus Wesselsbron, Nipah, de la fièvre de la vallée du Rift et de l'encéphalite japonaise [14,18,21]. La production de viande locale ne couvrant pas les besoins, il existe de plus une importation de viande congelée à partir de l'étranger [6]. Notons que l'importation de viande sur pied est interdite à La Réunion depuis plusieurs dizaines d'années, ce qui n'est pas le cas à Madagascar, à Mayotte et aux Comores, où des animaux sur pied sont importés illégalement à partir de l'Afrique.

\section{Susceptibilité de la population humaine}

La population réunionnaise est naïve sur le plan immunologique vis-à-vis de ces maladies, ce qui représente un facteur de risque majeur de déclenchement d'une flambée épidémique. 


\begin{tabular}{|c|c|c|c|c|c|c|}
\hline & Virus Sindbis & $\begin{array}{l}\text { Virus } \\
\text { de l'encéphalite } \\
\text { japonaise }\end{array}$ & Virus Wesselsbron & Virus Nipah & Virus West Nile & $\begin{array}{l}\text { Virus de la fièvre } \\
\text { de la vallée du Rift }\end{array}$ \\
\hline $\begin{array}{l}\text { Principaux } \\
\text { moustiques } \\
\text { vecteurs } \\
\text { (par genre) }\end{array}$ & $\begin{array}{l}\text { Culex } \\
\text { Aedes }++\end{array}$ & $\begin{array}{l}\text { Culex } \\
\text { Anopheles }\end{array}$ & Aedes & $\varnothing$ & Culex & $\begin{array}{l}\text { Culex } \\
\text { Aedes }++ \\
\text { Anopheles }\end{array}$ \\
\hline Réservoir naturel & $\begin{array}{l}\text { Petits mammifères } \\
\text { Oiseaux sauvages } \\
\text { migrateurs }\end{array}$ & $\begin{array}{l}\text { Oiseaux aquatiques } \\
\text { sauvages }\end{array}$ & Mammifères +++ & $\begin{array}{l}\text { Chauve-souris } \\
\text { du genre Pteropus }\end{array}$ & $\begin{array}{l}\text { Oiseaux sauvages } \\
\text { migrateurs }\end{array}$ & $\begin{array}{l}\text { Bétail (bovin, } \\
\text { mouton, chameau, } \\
\text { chèvres) }\end{array}$ \\
\hline Hôtes & $\begin{array}{l}\text { Humains } \\
\text { Mammifères }\end{array}$ & $\begin{array}{l}\text { Humains } \\
\text { Bétail ++ (porc) }\end{array}$ & $\begin{array}{l}\text { Mammifères } \\
\text { (moutons) } \\
\text { Humains }\end{array}$ & $\begin{array}{l}\text { Humains } \\
\text { Porcs }\end{array}$ & $\begin{array}{l}\text { Chevaux } \\
\text { Humains }\end{array}$ & $\begin{array}{l}\text { Ruminants } \\
\text { Humains }\end{array}$ \\
\hline $\begin{array}{l}\text { Conditions } \\
\text { propices } \\
\text { au développement }\end{array}$ & Climat & $\begin{array}{l}\text { Rizière (eau } \\
\text { stagnante) } \\
\text { Elevage intensif } \\
\text { porcin }\end{array}$ & Elevage intensif & $\begin{array}{l}\text { Consommation } \\
\text { d'aliment souillé } \\
\text { Transmission } \\
\text { interhumaine ++ }\end{array}$ & $\begin{array}{l}\text { Climat } \\
\text { Densité vectorielle }\end{array}$ & $\begin{array}{l}\text { Chaleur } \\
\text { Fortes pluies } \\
\text { Inondations++ }\end{array}$ \\
\hline Symptomatologie & $\begin{array}{l}\text { Polyarthralgies } \\
\text { Rash } \\
\text { Fièvre } \\
\text { Pas de décès }\end{array}$ & $\begin{array}{l}\text { Pathogénicité faible } \\
\text { ou } \\
\text { Méningo-enc } \\
\text { éphalite } \\
\text { décès : } 25-50 \%\end{array}$ & $\begin{array}{l}\text { Asymptomatique ou } \\
\text { Syndrome pseudo } \\
\text { grippal } \\
\text { Pas de décès }\end{array}$ & $\begin{array}{l}\text { Encéphalite aiguë } \\
\text { Coma } \\
\text { Décès : } 40-75 \%\end{array}$ & $\begin{array}{l}\text { Fièvre } \\
\text { Céphalée } \\
\text { Arthralgies } \\
\text { Décès }:<4 \%\end{array}$ & $\begin{array}{l}\text { Sd pseudo grippal } \\
\text { ou } \\
\text { Hépatite /méningo- } \\
\text { encéphalite } \\
\text { Décès : } 1 \%\end{array}$ \\
\hline $\begin{array}{l}\text { Répartition } \\
\text { géographique / } \\
\text { proximité avec La } \\
\text { Réunion }\end{array}$ & $\begin{array}{l}\text { Afrique du Sud } \\
\text { et de l'est, } \\
\text { Asie du Sud-Est } \\
\text { Australie, Europe } \\
\text { Madagascar }\end{array}$ & Asie du Sud-Est & $\begin{array}{l}\text { Afrique (Afrique } \\
\text { du Sud }+++ \text { ) } \\
\text { Madagascar }\end{array}$ & $\begin{array}{l}\text { Asie du Sud-Est, } \\
\text { Madagascar } \\
\text { Iles Mascareignes }\end{array}$ & $\begin{array}{l}\text { Afrique } \\
\text { Moyen-Orient } \\
\text { Asie du Sud Est } \\
\text { Australie, } \\
\text { Madagascar }\end{array}$ & $\begin{array}{l}\text { Afrique }+++ \\
\text { Moyen-Orient } \\
\text { Madagascar } \\
\text { Comores }\end{array}$ \\
\hline $\begin{array}{l}\text { Risques pour La } \\
\text { Réunion* }\end{array}$ & $\begin{array}{l}\text { Présence } \\
\text { de moustiques } \\
\text { vecteurs } \\
\text { Migration } \\
\text { de population } \\
\text { Climat }\end{array}$ & $\begin{array}{l}\text { Présence } \\
\text { de moustiques } \\
\text { vecteurs } \\
\text { Migration } \\
\text { de population } \\
\text { Rizières irriguée } \\
\text { à Madagascar }\end{array}$ & $\begin{array}{l}\text { Migration } \\
\text { de population } \\
\text { Elevages locaux }\end{array}$ & $\begin{array}{l}\text { Transmission } \\
\text { interhumaine } \\
\text { Présence du genre } \\
\text { Pteropus } \\
\text { dans la région }\end{array}$ & $\begin{array}{l}\text { Présence } \\
\text { des moustiques } \\
\text { vecteurs } \\
\text { Migration } \\
\text { de population } \\
\text { (oiseaux et homme) }\end{array}$ & Climat \\
\hline
\end{tabular}

\section{Un environnement propice}

\section{Présence de vecteurs}

Cinq des zoonoses précédemment citées sont des arboviroses transmises par les moustiques (le virus Nipah fait exception). Il existe à La Réunion douze espèces de moustiques appartenant à quatre genres différents. Les principales espèces de moustiques potentiellement vecteurs de pathologies sont regroupées dans le tableau 2.

Ces vecteurs sont également présents dans les autres îles des Mascareignes et la zone océan Indien, favorisant la dissémination d'un virus sur un territoire après qu'il ait été introduit par l'Homme ou un animal virémique. L'augmentation permanente et régulière des échanges aériens et mari- times entre tous ces pays sont autant de facteurs favorisant l'introduction d'agents auparavant absents.

Les virus West Nile, de l'encéphalite japonaise et Sindbis se transmettent par des moustiques du genre Culex (Tableau 2). Or, La Réunion n'a pas connu ces dernières années de circulation de maladies transmises par les Culex alors que c'est le cas dans d'autres régions de l'océan Indien. La possibilité d'importation de ces maladies ne peut donc être exclue.

\section{Des réservoirs de virus}

À La Réunion, il n'existe pas de population ornithologique importante (oiseaux sauvages ou migrateurs), population qui constitue habituellement un facteur de risque de 
Tableau 2 Espèces de moustiques présentes à La Réunion / Mosquito species present in Reunion Island.

\begin{tabular}{|c|c|}
\hline Espèces & $\begin{array}{l}\text { Pathologies majeures } \\
\text { transmises }\end{array}$ \\
\hline $\begin{array}{l}\text { Anopheles coustani } \\
\text { arabiensis }\end{array}$ & Paludisme* \\
\hline Aedes albopictus & $\begin{array}{l}\text { Dengue, Chikungunya, autres } \\
\text { arboviroses }\end{array}$ \\
\hline Aedes aegypti & $\begin{array}{l}\text { Dengue, Chikungunya, Zika, } \\
\text { autres arboviroses }\end{array}$ \\
\hline Culex quinquefasciatus & $\begin{array}{l}\text { Encéphalite japonaise, West } \\
\text { Nile }\end{array}$ \\
\hline Culex tritaeniorhynchus & $\begin{array}{l}\text { Encéphalite japonaise, West } \\
\text { Nile et autres arboviroses }\end{array}$ \\
\hline Culex univittatus & $\begin{array}{l}\text { Sindbis, Encéphalite } \\
\text { japonaise, West Nile }\end{array}$ \\
\hline Culex tigripes & Encéphalite japonaise \\
\hline
\end{tabular}

développement et de dissémination, notamment des virus West Nile [10], de l'encéphalite japonaise [23] et Sindbis [20]. Le déplacement du virus Sindbis d'un pays à l'autre par le biais des oiseaux a déjà été démontré, de la Suède à l'Afrique du Sud ou entre les différentes régions de l'Australie [20]. Aussi, même si La Réunion n'est pas une escale migratoire importante, il n'est pas exclu que des oiseaux migrateurs viennent à La Réunion, en raison notamment du réchauffement climatique qui pourrait modifier les trajets migratoires et favoriser ainsi l'introduction de ces virus.

La fièvre catarrhale ovine, encore appelée Blue Tongue, est enzootique à La Réunion, ainsi qu'une autre maladie très proche, la maladie épizootique hémorragique. Quelques cas de fièvre catarrhale ovine ont également été rapportés à l'Ile Maurice (données non publiées).

Les chauves-souris de l'espèce niger du genre Pteropus (Grande roussette des Mascareignes ou Roussette noire), réservoir naturel du virus Nipah [13], sont présentes en petit nombre à La Réunion [11]. Ce genre est également présent à Madagascar, aux Comores, aux Seychelles et à Maurice [20], soulignant ici le risque de la proximité géographique avec des régions touchées. Des traces de virus Nipah ont déjà été mises en évidence chez des chauves-souris à Madagascar, mais aucun cas humain n'y a été détecté.

En ce qui concerne les élevages d'animaux domestiques, une étude récente a montré qu'une introduction du virus de l'encéphalite japonaise sur la côte ouest des Etats-Unis est possible, là où les élevages de porcs et le vecteur cohabitent. Il existe en effet sur la côte ouest des Etats-Unis de nom- breuses fermes de production porcines naïves. Le transport du bétail sur des distances moyennes d'une ferme à l'autre et la coexistence d'oiseaux migrateurs pourraient y jouer un rôle critique dans la propagation du virus dans ces zones vierges [15].

À La Réunion bien qu'il n'y ait aucune importation de porcs sur pieds, des contacts avec des oiseaux migrateurs sont toujours possibles.

\section{La proximité avec des pays en zone d'endémie}

La proximité, tant physique (situation géographique) que maritime et aérienne (via le transport) avec des pays connus pour être des zones endémiques ou des zones où les virus ont été mis en évidence par des enquêtes de séroprévalence (Sindbis, FVR et Wesselsbron en Afrique du Sud, Nipah, Wesselsbron présent uniquement chez les animaux, Sindbis, FVR et West Nile à Madagascar [5], FVR aux Comores [19] et à Mayotte [22], encéphalite japonaise en Asie du Sud-Est et en Australie...) fait de La Réunion une zone à risque d'introduction de ces virus.

Si le transport d'animaux ou de viande est un facteur de risque majeur de circulation de ces zoonoses entre les territoires, il ne faut pas écarter pour autant une possible propagation par l'Homme. Ainsi, par exemple, l'hypothèse envisagée pour expliquer l'épidémie de virus Zika en Micronésie en 2007 est l'importation d'un cas humain depuis les Philippines [7].

En 2011, l'aéroport principal de La Réunion a accueilli près de 1900000 voyageurs [2]. Les vols étaient en provenance ou à destination directe de France métropolitaine, d'Australie, de Nouvelle-Calédonie, de Thaillande, d'Afrique $\mathrm{du}$ Sud et des îles du sud-ouest de l'océan Indien (SOOI). Certains voyageurs étaient en transit en provenance d'autres pays (Inde, Malaisie, Indonésie, Cambodge, Vietnam, Kenya, Tanzanie).

Environ 760 cargos ont transité par Le Port de La Réunion en 2011, en provenance pour moitié des îles du SOOI (Madagascar particulièrement), mais également d'Asie (Malaisie, Vietnam), d'Afrique (Kenya, Mozambique, Afrique du Sud) ou encore d'Amérique du Sud (Brésil, Uruguay).

\section{Les conditions climatiques}

Le climat tropical et les changements climatiques actuels sont favorables à l'importation et à l'émergence de nouveaux virus. À titre d'exemple, on peut citer la provenance des chauves-souris du genre Pteropus à La Réunion, vraisemblablement importées de l'île Maurice par un cyclone en 2008 (données non publiées) ou encore les trajets des oiseaux migrateurs perturbés par les courants chauds. 
Un autre exemple réside dans les foyers de fièvre de la Vallée du Rift (FVR) qui dépendent totalement des conditions climatiques. Ni annuels, ni saisonniers, ces foyers apparaissent de façon cyclique en fonction de la pluviométrie et nécessitent des précipitations supérieures à la moyenne associées à des inondations prolongées, favorisant ainsi la prolifération conséquente des populations de moustiques vecteurs. Ce scénario concerne uniquement les épidémies majeures de FVR en Afrique de l'Est et australe. Toutefois, la dangerosité du virus de la FVR, qui est capable de s'adapter à des conditions écologiques très variées et qui est déjà présent dans la région (Afrique de l'Est, Madagascar, Mayotte), ne met pas La Réunion à l'abri.

De plus, la stratégie du virus FVR qui consiste en sa transmission trans-ovarienne chez certaines espèces d'Aedes, peut permettre aux œufs infectés de rester viables dans la boue sèche d'un épisode d'inondation à l'autre, comme c'est actuellement le cas au Kenya depuis 1997 [17]. Toutefois, cette stratégie n'a été démontrée uniquement que lors des épidémies majeures de FVR en Afrique de l'Est et Australe.

\section{Agent étiologique}

\section{Présence des virus}

Hormis le virus West Nile dont les traces sérologiques ont été retrouvées chez des chevaux à La Réunion (données CIRAD - CRVOI, non publiées), les autres virus préalablement cités n'ont jamais été mis en évidence. Néanmoins, leur présence ne peut être exclue car aucune enquête de prévalence, en particulier animale, n'a été réalisée à ce jour.

\section{Conclusion}

À La Réunion, les trois facteurs d'accroissement du risque d'émergence : un environnement favorable, un hôte susceptible, et la présence potentielle d'un agent étiologique, sont présents, eu égard à des conditions climatiques changeantes, à une modification des comportements humains ou encore à l'importation possible et inopinée d'un agent ou d'un vecteur par voie maritime ou aérienne.

Les principaux risques d'émergence et de propagation sont représentés par les virus Sindbis et l'encéphalite japonaise pour lesquels tous les facteurs épidémiologiques (présence des vecteurs et des réservoirs/hôtes intermédiaires, facteurs climatiques, environnementaux et humains) sont réunis pour générer une épidémie. Si le virus Sindbis est peu virulent, une épidémie d'encéphalite japonaise serait en revanche d'une gravité bien supérieure en raison du taux de mortalité et des séquelles associées. Néanmoins, l'existence d'un vaccin efficace pourrait permettre sa prévention.
Il faut donc souligner toute l'importance de la surveillance de ces maladies. C'est pourquoi, à La Réunion, la Cire océan Indien suit en continu les tendances des maladies infectieuses à potentiel émergent et identifie les situations sanitaires inhabituelles et à risque pour le territoire. À une échelle plus large, le réseau de surveillance épidémiologique et de gestions des alertes (SEGA) de la Commission océan Indien permet l'échange d'informations et de données épidémiologiques entre les cinq pays du sud-ouest de l'océan Indien. Enfin, à l'image du réseau SEGA, le réseau AnimalRisk-OI [8] est chargé de la surveillance vétérinaire au sein des pays de la zone océan Indien.

Seule la réalisation d'enquêtes de séroprévalence chez l'Homme permettrait de connaître l'état immunitaire de la population réunionnaise envers ces pathologies. De même, des enquêtes de séroprévalence régulières chez les populations animales réservoirs, voire la constitution de populations animales sentinelles, en particulier chez les chauvessouris et les porcs, permettraient de repérer la présence et la propagation de telles pathologies. Outre la prise en compte de la manipulation délicate et dangereuse selon l'agent, requérant des laboratoires hautement spécialisés, présents à La Réunion (P3 humain, animalerie P3), c'est une solution qui pourrait permettre d'anticiper la transmission des pathologies émergentes de l'animal à l'Homme, voire une épidémie humaine. À la suite de l'épidémie de Chikungunya de 2005-2006, La Réunion a accru ses capacités de diagnostic et de réponse aux épidémies, notamment par la création du Centre régional de recherche et de veille de l'océan Indien, le développement des capacités des laboratoires du CHU, ainsi qu'en tissant des liens étroits avec les CNR de métropole et des liens scientifiques au plan international.

Conflit d'intérêt : les auteurs déclarent ne pas avoir de conflit d'intérêt.

\section{Références}

1. Bondre VP, Sapkal GN, Yergolkar PN, et al (2009) Genetic characterization of Bagaza virus (BAGV) isolated in India and evidence of anti-BAGV antibodies in sera collected from encephalitis patients. J Gen Virol 90(Pt 11):2644-9. Epub 2009 Jul 1

2. Chambre de commerce et d'industrie de La Réunion (2010) Rapport annuel de l'aéroport de La Réunion, Roland Garros.

3. Calzolari M, Gaibani P, Bellini R, et al (2010) Mosquito, bird and human surveillance of West Nile and Usutu viruses in EmiliaRomagna Region (Italy) in 2010. PLoS One 7(5):e38058. Epub 2012 May 30

4. Cao Z, Zhang C, Liu Y, et al (2011) Tembusu virus in ducks, China. Emerg Infect Dis 17 (10):1873-5

5. Cardinale E, Roger M, Elissa N, et al (2011). Le réseau régional AnimalRisk dans l'Océan Indien. Bulletin épidémiologique de l'Anses 
6. Direction de l'alimentation, de l'agriculture et de la foret de la Réunion (DAAF) source : http://www.daf974.agriculture.gouv.fr/ [consulté en avril 2012]

7. Duffy MR, Chen TH, Hancock WT, et al (2009) Zika Virus Outbreak on Yap Island, Federated States of Micronesia. N Engl J Med 360(24):2536-43

8. Field H, Schaaf K, Kung N, et al (2010) Hendra virus outbreak with novel clinical features, Australia. Emerg Infect Dis 16 (2):338-40

9. Haut conseil de la santé publique (2010) Les maladies infectieuses émergentes : état de la situation et perspective. La documentation française

10. Hubálek Z, Halouzka J (1999) West Nile fever -a reemerging mosquito-borne viral disease in Europe. Emerg Infect Dis 5 (5):643-50

11. Museum and Institute of Zoology of the Polish Academy of Sciences - Bat distribution viewer (http://ims.cartoninjas.net/thebatsgmaps/)

12. Kono Y, Tsukamoto K, Abd Hamid M, et al (200) Encephalitis and retarded growth of chicks caused by Sitiawan virus, a new isolate belonging to the genus Flavivirus. Am J Trop Med Hyg 63(1-2):94-101

13. Iehlé C, Razafitrimo G, Razainirina J, et al (2007) Henipavirus and Tioman virus antibodies in pteropodid bats, Madagascar. Emerg Infect Dis 13(1):159-61

14. Mills JN, Alim AN, Bunning ML, et al (2009) Nipah Virus Infection in Dogs, Malaysia. Emerg Infect Dis 15(6):950-2
15. Nett RJ, Campbell GL, Reisen WK (2009) Potential for the emergence of Japanese encephalitis virus in California. Vector-Borne Zoonotic Dis 9(5):511-7

16. Omran A (1971). The epidemiologic transition. A theory of the epidemiology of population change. Milbank Mem Fund Q 49 (4):509-38

17. Organisation mondiale de la santé animale (2007) Le point sur la fièvre de la vallée du Rift. Bull de l'OIE (2):39-40

18. Pfeffer M, Dobler G (2010) Emergence of zoonotic arboviruses by animal trade and migration. Parasit Vectors 3(1):35

19. Roger M, Girard S, Faharoudine A, et al (2011). Rift Valley Fever in ruminants, Republic of Comoros, 2009. Emerg Infect Dis 17(7):1319-20

20. Sammels LM, Lindsay MD, Poidinger M, et al (1999) Geographic distribution and evolution of Sindbis virus in Australia. J Gen Virol 80(Pt 3):739-48

21. The Center for Food Security and Public Health (2006) [Wesselsbron disease http://www.cfsph.iastate.edu/Factsheets/pdfs/wesselsbron.pdf]

22. Tortosa P, Pascalis H, Guernier V, et al (2012) Deciphering arboviral emergence within insular ecosystems. Infect Genet Evol. 12 (6):1333-9. Epub 2012 Apr 4

23. Weaver SC, Powers AM, Brault AC, Barrett AD (1999) Molecular epidemiological studies of veterinary arboviral encephalitides. Vet J 157(2):123-38 